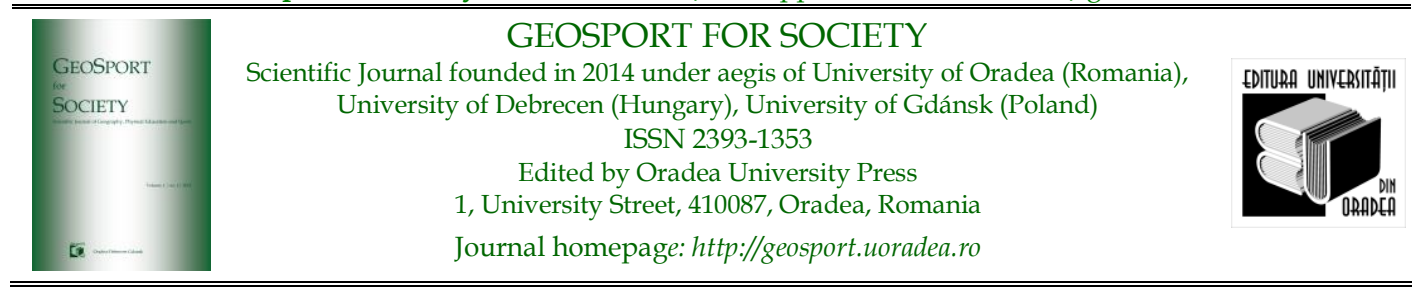

\title{
Linear kinematic analysis of cinematic biomechanics in semi- squat knee flexion: a case study
}

\author{
Dan Alexandru SZAB0 ${ }^{1 *}$, Nicolae NEAGU ${ }^{1}$, Andreea ILIEȘ ${ }^{1}$, \\ Mariana ARDELEAN ${ }^{1}$
}

\begin{abstract}
1. George Emil Palade University of Medicine, Pharmacy, Science, and Technology, Romania, Address: Str. Gheorghe Marinescu, No. 38, C.P. 540139, Târgu Mureș, Romania, e-mails: dan-alexandru.szabo@umfst.ro,

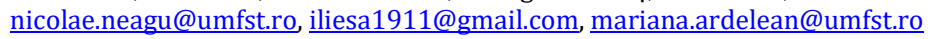

* Corresponding author

Citation: Szabo, D.A., Neagu, A., Ilieș, A., \& Ardelean, M. (2021). Linear kinematic analysis of cinematic biomechanics in semi-squat knee flexion: a case study. Geosport for Society, 14(1), 56-66. https://doi.org/10.30892/gss.1406-073

Article history: 19.05.2021; Revised: 10.06.2021; Accepted: 25.06.2021, Available online: 30.06 .2021

\begin{abstract}
The study emphasized the necessity of kinesiology and biomechanical movement analysis in today's physical education, kinesiotherapy, and sports. Our experiment observed a biomechanical motion configuration of semi-squat knee flexion, vertical position, angle, speed, and acceleration. The Kinovea software, version 0.9.3, is utilized as a study tool for biomechanical investigation employing some specific kinesiological characteristics of the movement. The findings of the biomechanical action emphasized the unique semi-squat knee flexion and extension, displaying the complete action from a certain linear angle, angular velocity, and acceleration.
\end{abstract}

Keywords: biomechanical movement, kinesiology, semi-squat knee flexion

\section{Introduction}

Angular and linear kinetics and kinematics can be accomplished using the inverse dynamics method described by Whittlesey and Robertson (2004) as 'the mechanism by which forces and moments of force are indirectly determined by the kinematics and inertial properties of moving bodies (Sanders et al., 2016). The center of mass (CM) location can be accomplished by modeling the body as a set of rigid connections (Sanders et al., 2016). Linear and angular momentum can be extracted from linear and angular displacements concerning the digitized coordinates' reference axes. Angular moments of the body segments concerning each axis are then calculated, and the net torque of each axis is derived as derivatives of the entire body angular momentum of each axis (Sanders et al., 2016). 
The inverse dynamic method can also help swimming research provide quantitative knowledge on linear and angular motion (Sanders et al., 2016).

A modern kind of prosthetic management has newly gone recommended position management (Geethanjali, 2016). This supports on the findings of past dimensionality-decrease experiments conducted on hand kinematics (Santello et al., 1998; Todorov and Ghahramani, 2004; Ingram et al., 2008; Portnova-Fahreeva et al., 2020). In some experiments, the Principal Component Analysis was used to simplify complex hand grip kinematics by discovering a decreased proportion of linear input signal combinations that describe much of the heterogeneity found in data capture. These variations cover a latent variety of kinematics (Portnova-Fahreeva et al., 2020). The sports practices will enhance skills (Sopa, 2015; Sopa, 2019a; Sopa, 2019b), sustain wellbeing, build community harmony and connectivity (Sopa, 2014), and facilitates the learning of correct movement from a biomechanical point of view.

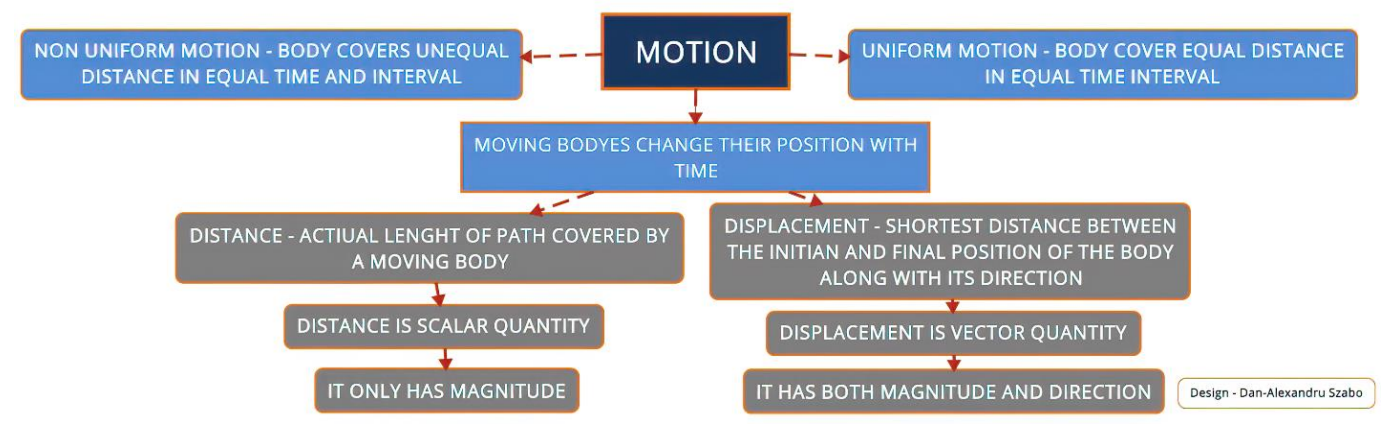

Figure 1. The human motion

HUMAN MOVEMENT ANALYSIS

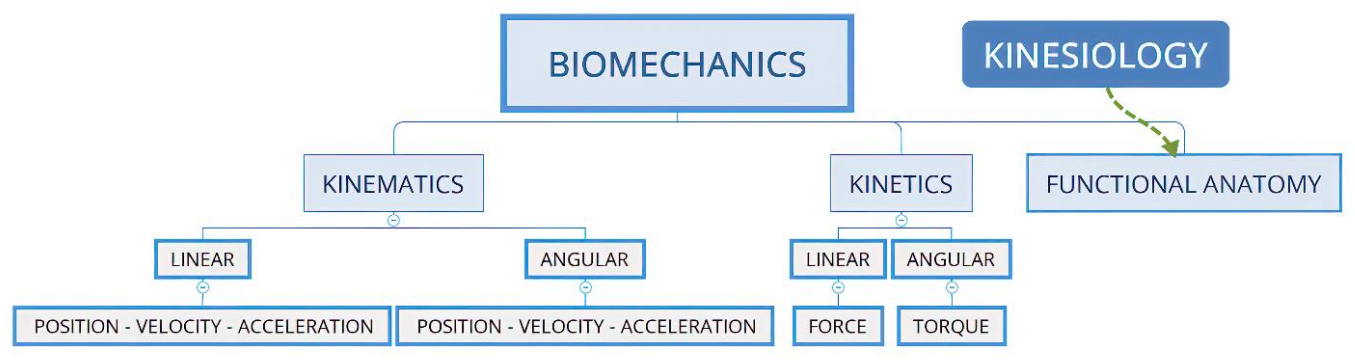

Hamill et al., $2003 \ominus$ Design: Dan-Alexandru Szabo

Figure 2. Human movement analysis

(Source: Hamill et al, 2003)

Traditionally, three types of data are known to explain human movements fully: kinematic, kinematic, and electromyographic (EMG) (Winter, 1991; Lencioni et al., 2019). Kinematic data include displacement and alignment of body parts, joint angles, 
and spatial-temporal gait parameters. Kinetic data include ground reaction forces (GRF), mechanic moments, lower limb powers, kinetic and potential energy (Lencioni et al., 2019). Muscle activation patterns are studied by electrical signals (EMGs) consistent with muscle fiber contraction, which can be recorded non-invasively by surface electrodes connected to the skin over the muscle belly (Lencioni et al., 2019).

Sports biomechanics is usually practiced employing wearable detectors which empower non-invasive data acquisition during movements (Taborri et al., 2016). Moreover, wearable detectors allow athletic activity to be conducted in the biological world, overwhelming the ecological constraint of laboratory experiments, such as the use of the optoelectronic 3D device, nevertheless evaluated to be the golden criterion for movement evaluation (Tabori et al., 2016; van der Kruck and Reijne, 2018).

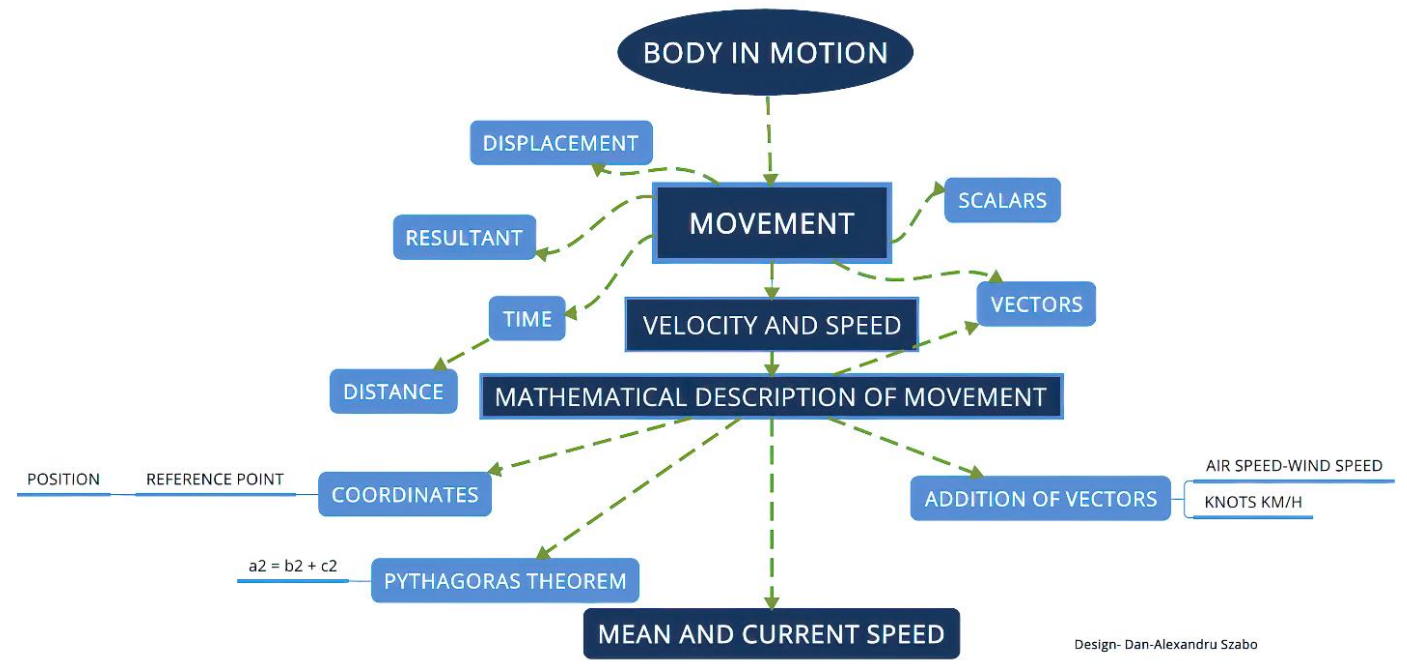

Figure 3. The body movement

Inertial sensors (Kos and Umek, 2019; Kinnunen et al., 2019; Gopfert et al., 2017), force sensors (Lee et al., 2017; Buckeridge et al., 2015; Kos and Umek, 2018a), and electromyography sensors (Brochner et al., 2018; Cruz Ruiz et al., 2015) appear frequently utilized for objective and covert quantification of kinematics, kinetics, and muscle movement during sports events. One exciting path for wearable sensor usage is the real-moment biofeedback processes (Kos and Umek, 2018b) that can give improved input knowledge to athletes and/or coaches at the same time (Kos and Umek, 2019; Umek and Kos, 2016; Kos et al., 2019). Lee et al. (Lee et al., 2017) found that hip, knee, and ankle joint forces and moments, measured based on a conventional inverse dynamic study using motion capture data and the ground response force, were higher mid-turn than for short-turn ski carving. Purevsuren et al., (Purevsuren et al., 2018) concluded that short trackers had intense internal rotational moments when the knee was flexed. However, this conclusion may not be accurate because pressure insoles can only approximate the usual force component on the plantar surface and hence the moment (free moment) and force components parallel to the plantar surface. Instead, horizontal plane forces are essential for short-track speed skating (van der Kruk et al., 
2018). In comparison, only short skating was used in the study when skaters are either approaching, leaving, or within a curve (van der Kruk et al., 2018). Applications of surface electromyography (SEMG) in sports science have been increasingly widespread and diversified over the last decade (Merletti and Muceli, 2019). Thanks to wireless networks' emergence, SEMG is now primarily used as a descriptive instrument and in quantitative studies. Bipolar (i.e. using a series of two electrodes) setups are widespread in sports science to record non-invasive summation of action potentials through the skin, giving an analog signal as output that defines the electrical potential difference (voltage) measured between the two electrodes (Merletti and Muceli, 2019).

\section{Methodology}

\section{Study Design and Subjects}

The study methodology was clarified, and informed consent was received from the subject to interpret the findings and publish them. Both activities have been carried out following the specifications of the Helsinki Declaration.

This thesis focuses on the premise of using Kinovea, version 0.9.3, kinetic, and biomechanical analysis program, and we can strengthen the teaching method in biomechanics and kinesiology (Szabo et al., 2020).

The case study analyzes the leg's biomechanical linear movement on a student's semi-squat knee flexion. The student was in the first term at the Master's curriculum Physical Therapy and Functional Rehabilitation at "G. E. Palade" University of Medicine, Pharmacy, Science, and Technology from Târgu Mureș, Romania. The objective of this experiment appears to emphasize the circumstance that specialized biomechanical measurements, such as linear kinematics, can exclusively be obtained through the aid of videotape analysis tools minus any additional mechanisms of assistance (Szabo et al., 2020).

Both assessments were transported abroad in the practical workshop in Biomechanics and Kinesiology from 16-September 2020 to 28-September 2020 at the headquarters of the Discipline of Human Movement Sciences (Szabo et al., 2020).

\section{Procedure}

The testing technique involved multiple trials of execution of semi-squat knee flexion and was reported as the strongest and accurate scientific execution. The program used to analyze the movement of the subject was Kinovea, version 0.9.3.

Table 1. Linear kinematics - from frame 0 to frame 2568

\begin{tabular}{ccccccc}
\hline \multirow{2}{*}{ Time (ms) } & \multicolumn{3}{c}{ SPEED } & \multicolumn{3}{c}{ ACCELERATION } \\
\cline { 2 - 7 } & Angle 1 - o & Angle 1 - a & Angle 1 - b & Angle 1 - o & Angle 1 - a & Angle 1 - b \\
\hline 0 & - & - & - & - & - & - \\
33 & 14.4588089 & 19.88516617 & 20.06421852 & - & - & -49.3273735 \\
67 & 13.91840935 & 18.05595398 & 17.57689857 & -16.50285912 & -55.76958466 & -68.76911163 \\
133 & 12.87868881 & 14.6063633 & 15.55068398 & -26.75684929 & -44.2489357 & -108.4627838 \\
167 & 11.57306957 & 13.21319866 & 12.1863184 & -45.64212036 & -42.52270126 & -72.73350525 \\
200 & 9.834025383 & 11.76978779 & 8.315422058 & -31.9717617 & -41.17425156 &
\end{tabular}


DOI 10.30892/gss.1406-073

\begin{tabular}{|c|c|c|c|c|c|c|}
\hline 267 & 10.49862099 & 9.806860924 & 8.878773689 & 37.76114273 & -8.549201965 & 49.04633331 \\
\hline 300 & 11.9592638 & 9.896280289 & 10.60621071 & 22.76117897 & 5.938308716 & 39.62181854 \\
\hline 333 & 12.01695824 & 10.20298958 & 11.52183914 & -25.6070919 & 4.003691673 & 21.79558563 \\
\hline 400 & 8.318258286 & 9.666053772 & 11.81884766 & -42.19184113 & -16.22542381 & -24.61861038 \\
\hline 433 & 7.436578751 & 9.081001282 & 10.41789436 & -12.46854496 & -11.00447941 & -22.66716576 \\
\hline 467 & 7.486515045 & 8.931974411 & 10.30678177 & 14.32147217 & 4.285830975 & 19.49443817 \\
\hline 533 & 9.998731613 & 10.00110817 & 11.16951084 & 45.38505554 & 15.63881397 & -45.73445892 \\
\hline 567 & 11.41944122 & 10.41012096 & 8.667492867 & 41.42559814 & 8.368052483 & -61.88111115 \\
\hline 600 & 12.76212215 & 10.55931854 & 7.04158783 & 46.51765823 & 2.498581171 & -15.22095871 \\
\hline 667 & 15.52557755 & 10.40012455 & 15.24921608 & -12.23161125 & -8.871285439 & 468.5710754 \\
\hline 700 & 13.70657158 & 9.985014915 & 38.90926361 & -100.9677048 & -12.24093246 & 953.4970093 \\
\hline 733 & 8.79029274 & 9.583564758 & 78.85444641 & -101.2564087 & -6.708684444 & 1373.266724 \\
\hline 800 & 9.644400597 & 10.06261921 & 186.4218597 & 6.18184042 & 28.05942345 & 1609.255493 \\
\hline 833 & 7.364401817 & 11.40926552 & 237.8653259 & 52.57108307 & 56.15736008 & 1351.076538 \\
\hline 867 & 13.15127659 & 13.80872631 & 276.5485535 & 353.4708862 & 86.25759888 & 916.4918823 \\
\hline 933 & 50.93548584 & 21.01438332 & 310.6651001 & 604.8991699 & 113.4147415 & 332.1832886 \\
\hline 967 & 71.29470062 & 24.7288723 & 321.1611023 & 593.0803833 & 100.9663849 & 349.0402222 \\
\hline 1002 & 90.49829102 & 27.74958038 & 333.9486389 & 525.3041992 & 76.14069366 & 348.1257324 \\
\hline 1068 & 118.5794907 & 30.91671181 & 348.7219543 & 320.1601563 & 21.49717712 & 83.69590759 \\
\hline 1102 & 127.6933594 & 31.242033 & 349.9667664 & 194.1765137 & 1.975132942 & 69.21057129 \\
\hline 1135 & 131.532486 & 31.04846764 & 353.3388062 & -9.88407135 & -8.989545822 & 161.7615509 \\
\hline 1202 & 115.1553726 & 30.28544426 & 370.3468323 & -376.0195923 & -6.385743141 & 252.4327393 \\
\hline 1235 & 101.9507599 & 30.2163887 & 377.5965576 & -310.9258118 & 5.826210499 & 133.3746338 \\
\hline 1268 & 94.41434479 & 30.67409515 & 379.2438965 & -91.56030273 & 20.87622833 & 34.03640366 \\
\hline 1335 & 104.5520248 & 32.47192383 & 389.2814026 & 293.887146 & 15.65455437 & 426.9640503 \\
\hline 1368 & 115.4474411 & 32.65325928 & 408.3486633 & 266.2252197 & -8.261001587 & 550.5096436 \\
\hline 1402 & 122.3111954 & 31.92085457 & 426.004425 & 77.00702667 & -35.59354019 & 361.1493835 \\
\hline 1468 & 109.9370422 & 27.80055618 & 424.9711304 & -402.7821045 & -82.52157593 & -419.5175781 \\
\hline 1502 & 93.71585846 & 24.77411652 & 404.4550781 & -509.6691589 & -89.65662384 & -787.5653687 \\
\hline 1535 & 75.93837738 & 21.81980324 & 372.4347534 & -505.2731323 & -77.10263824 & -1124.958008 \\
\hline 1602 & 47.93202209 & 18.37613678 & 275.2306824 & -323.6434326 & -33.48536301 & -1786.376831 \\
\hline 1635 & 38.42105484 & 17.3970871 & 210.2477264 & -267.2239075 & -38.91363525 & -2096.359375 \\
\hline 1668 & 30.10623169 & 15.78031254 & 135.3881683 & -192.6002197 & -62.85666656 & -2362.535645 \\
\hline 1735 & 25.50746727 & 10.24510956 & 39.42596436 & -12.38614082 & -79.69115448 & 1098.280151 \\
\hline 1768 & 24.7469635 & 7.888103962 & 125.9126282 & -33.15065765 & -58.85387802 & 2490.628418 \\
\hline 1802 & 23.29607582 & 6.319125175 & 205.5691071 & 110.7941589 & -49.75124741 & 2219.383545 \\
\hline 1868 & 52.61846161 & 3.383494377 & 330.6090698 & 659.7818604 & 35.57683945 & 1525.462524 \\
\hline 1902 & 76.15002441 & 6.942567348 & 375.721283 & 680.927063 & 142.7825623 & 1175.789185 \\
\hline 1935 & 98.04128265 & 12.90813637 & 409.0428162 & 637.3983154 & 186.5229492 & 819.1052856 \\
\hline 2002 & 141.1400299 & 26.00279808 & 440.5660706 & 724.3531494 & 203.0322723 & 171.5869598 \\
\hline
\end{tabular}


vol. 14 , no. 1,2021 , pp. $56-66$

\begin{tabular}{lcccccc}
\hline \hline 2035 & 166.9888153 & 32.9287529 & 441.8077087 & 794.690918 & 210.7038116 & -60.0773468 \\
2068 & 194.1517334 & 40.05828476 & 436.5584717 & 780.5303955 & 208.7981262 & -223.8317108 \\
2135 & 238.3856354 & 52.79893112 & 413.3502197 & 447.4147644 & 158.6675262 & -490.3284302 \\
2168 & 248.9017487 & 57.44140244 & 394.1679993 & 146.3161926 & 112.2802963 & -668.7575073 \\
2202 & 248.1459961 & 60.28884888 & 368.7391968 & -194.8187103 & 52.16920853 & -758.1934814 \\
2268 & 215.0220337 & 59.12810516 & 330.7597046 & -681.0819092 & -89.96979523 & -81.02420044 \\
2302 & 190.4727631 & 54.91982651 & 338.1860352 & -734.1412354 & -158.1170044 & 480.055603 \\
2335 & 166.0494385 & 48.58054352 & 362.7829285 & -734.6373901 & -215.2737122 & 799.817749 \\
2402 & 112.7065735 & 31.14025688 & 408.4132996 & -988.630127 & -300.6525574 & 148.2341919 \\
2435 & 75.51820374 & 20.50376701 & 401.4280396 & -1223.613892 & -325.2480164 & -613.8639526 \\
2468 & 31.08256721 & 9.443833351 & 367.4640808 & -806.2472534 & -268.9533386 & -1269.656372 \\
2535 & 55.64744186 & 8.405345917 & 271.4120178 & - & - & - \\
2568 & - & - & - & - & - & -
\end{tabular}

\section{Results}
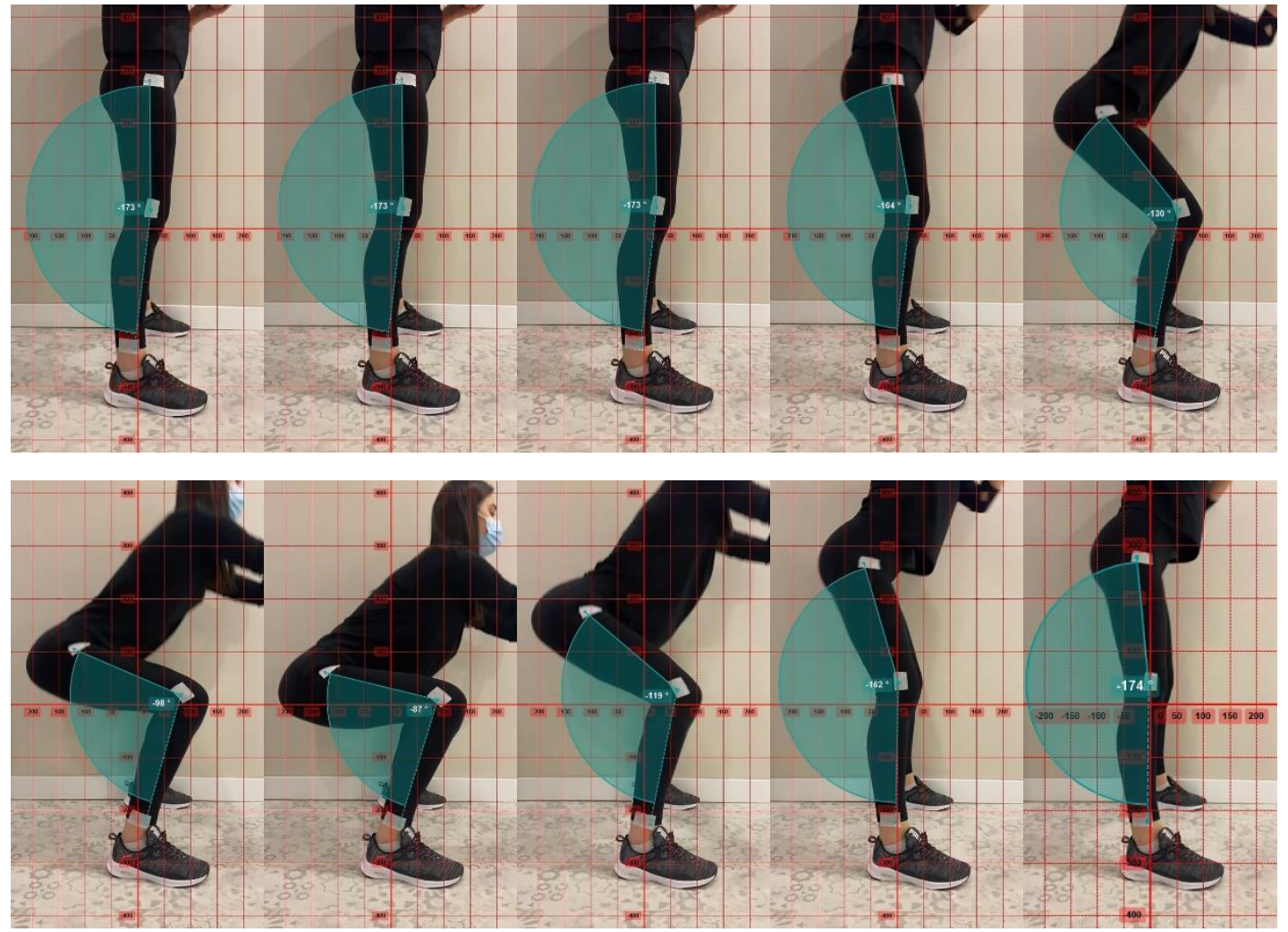

Figure 4. Graphical representation of linear variation in the semi-squat knee flexion 

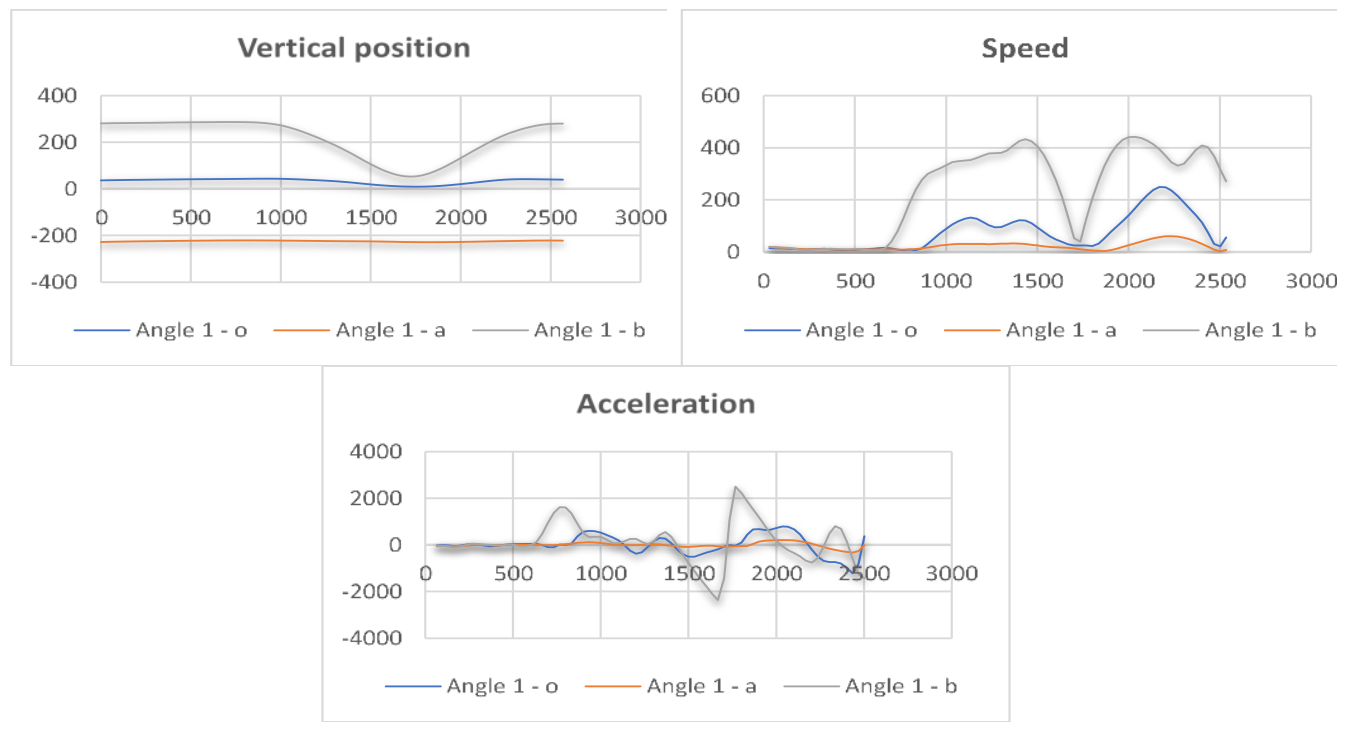

Figure 5. Representation of vertical position, speed and acceleration in the semi-squat knee flexion

\section{Discussion}

The purpose of this study was to investigate the linear kinematics and kinetics extracted from digital video. This complements the work of Sanders et al. (2015) and Szabo et al. (2020) to determine the reliability of linear and angular kinematics and kinetics. Although several systematic analyses already available in the literature have shown the efficiency, relevance, and utility of inertial sensors for sports applications (de Magalhaes et al., 2014; Johnston et al., 2019; Camomilla, et al., 2015), an outline of particular applications that can be applied by evaluating kinematics, kinetics, muscle function, and physiological parameters via a wearable. Our research highlighted the importance and viability of kinematic analysis in discovering the mechanism of lower member movement. Other scientific research also highlighted the importance of informatics programs in sports (Szabo et al., 2019) and preventing body dysfunctions (Sopa et al., 2019).

The evaluation of motor performance in sport is becoming more critical due to strengthening competitiveness and financial incentives among athletes. Wearable devices can provide crucial training and competitive success results. Among other sensor options, inertial sensors are the most commonly used, while force measurement devices and electromyography allow more knowledge on kinetics, and related muscle activity levels may offer further insight into the motor actions of athletes (Taborri et al., 2020).

The forces created by an athlete will provide helpful insight into their future success and risk of injury. Variables based on stand-alone force measures include the pressure center (CoP) (Buckeridge et al., 2015), the force path as a surrogate indicator of performance (Kinoshita et al., 2017), and the impact powers (Saponara, 2017). In 
conjunction with kinematic tests, force data were used to approximate mechanical capacity (van der Kruk et al., 2018), joint kinetics (Lee et al., 2017; Purevsuren et al., 2018), and muscle intensity (Urbanczyk, 2019).

The effect of workload and cadence on muscle coordination is essential, as muscle coordination has an impact on mechanical performance (Blake et al., 2012; Wakeling et al., 2010) and strength production (Dorel et al., 2012; Samozino et al., 2007; Wakeling et al., 2010), but few studies have looked at spatiotemporal muscle excitation-workloadtraining relationships across multiple muscles.

In an ice hockey kinematic analysis, scientists (Buckeridge et al., 2015) found that joint kinematics and plantar intensity application techniques remained remarkably stable through trials for a given subject. This was predicted during cyclical events such as running. Muscle function is customarily adjusted to retain the preferred direction of motion, i.e., maintaining joint angle trajectories (Nigg, 2001). Excellent reliability was observed for joint kinematics and plantar strength tests, while overall waveform intensity EMG showed moderate to an excellent agreement for each of the five measured muscles (Buckeridge et al., 2015).

Effective coaching results may be accompanied by proper and prompt input to the athlete on goal success shortcomings (Camomilla et al., 2015). Systematic, analytical, and accurate performance measurement and assessment, carried out using qualitative and quantitative analysis of mechanical variables that decide performance, will improve the correlation between research and coaching practices, especially in elite sports. An alternative to classical laboratory-based assessment is the use of magneto and inertial sensors that can calculate movement-related data, linear and angular motion, without room constraints and bulky setup (Armstrong et al., 2007, Dellaserra, 2014). The latest generation of inertial sensors is compact, low-cost, easy-to-use, and allows exercises to be carried out during training or competition, opening new insights in sports sciences. The use of wearable inertial sensors has recently been analyzed in swimming (de Magalhaes et al., 2014), running (Norris, 2014), and strength and ballistic evaluation (Mc Master et al., 2014).

\section{Conclusions}

The research theory has been verified, and, using Kinovea, version 0.9.3, kinetic, and biomechanical analysis program, the teaching method in the practical work of the Biomechanics and Kinesiology discipline has been strengthened. The students' input was often encouraging and, by using Kinovea software alone, they were able to put into reality the ideas accumulated in the course, the notions of biomechanics, kinesiology, and linear kinematics (acceleration, speed, and vertical position).

\section{References}

Armstrong, S. (2007). Wireless connectivity for health and sports monitoring: a review. Br J Sports Med, 41(5), 285-289.

Blake, O.M., Champoux, Y., \& Wakeling, J.M. (2012). Muscle coordination patterns for efficient cycling. Med Sci Sports Exerc, 44, 926-938. 
Brøchner, N.N., Hug, F., Guével, A., Colloud, F., Lardy, J., \& Dorel, S. (2018). Changes in motor coordination induced by local fatigue during a sprint cycling task. Medicine and Science in Sports and Exercise, 50(7), 1394-1404. https://doi.org/10.1249/MSS.0000000000001572.

Buckeridge, E., Levangie, M.C., Stetter, B., Nigg, S.R., \& Nigg, B.M. (2015). An on-ice measurement approach to analyse the biomechanics of ice hockey skating. PLoS One, 10(5), e0127324. https://doi.org/10.1371/journal.pone.0127324.

Camomilla, V., Bergamini, E., Fantozzi, S., \& Vannozzi, G. (2015). In-field use of wearable magnetoinertial sesnors for sports performance evaluation. 33rd International Conference on Biomechanics in Sports; 1425-1428.

Cruz Ruiz, A.L., Pontonnier, C., Sorel, A., \& Dumont, G. (2015). Identifying representative muscle synergies in overhead football throws. Computer Methods in Biomechanics and Biomedical Engineering, 18(sup1):1918-1919. https://doi.org/10.1080/10255842.2015.1070581.

de Magalhaes, F.A., Vannozzi, G., Gatta, G., \& Fantozzi, S. (2014). Wearable inertial sensors in swimming motion analysis: a systematic review. Journal of Sports Sciences, 33(7), 732-745. https://doi.org/10.1080/02640414.2014.962574.

Dellaserra, C.L. (2014). Use of integrated technology in team sports: a review of opportunities, challenges, and future directions for athletes. J Strength Cond Res, 28(2), 556-73.

Dorel, S., Guilhem, G., Couturier, A., \& Hug, F. (2012). Adjustment of muscle coordination during an allout sprint cycling task. Med Sci Sports Exerc, 44, 2154-2164.

Göpfert, C., Pohjola, M.V., Linnamo, V., Ohtonen, O., Rapp, W., \& Lindinger, S.J. (2017). Forward acceleration of the centre of mass during ski skating calculated from force and motion capture data. Sports Engineering, 20(2), 141-153. https://doi.org/10.1007/s12283-016-0223-9.

Hamill, J., \& Knutzen, K.M. (2006). Biomechanical basis of human movement. Lippincott Williams \& Wilkins.

Ingram, J.N., Körding, K.P., Howard, I.S., \& Wolpert, D.M. (2008). The statistics of natural hand movements. Exp. Brain Res. 188, 223-236. https://doi.org/10.1007/s00221-008-1355-3

Johnston, W., O'Reilly, M., Argent, R., \& Caulfield, B. (2019). Reliability, validity and utility of inertial sensor systems for postural control assessment in sport science and medicine applications: a systematic review. Sports Medicine, 49(5),783-818. https://doi.org/10.1007/s40279-019-01095-9.

Kinnunen, H., Häkkinen, K., Schumann, M., Karavirta, L., Westerterp, K. R., \& Kyröläinen, H. (2019). Training-induced changes in daily energy expenditure: methodological evaluation using wrist-worn accelerometer, heart rate monitor, and doubly labeled water technique. PLoS One, 14(7), e0219563. https://doi.org/10.1371/journal.pone.0219563.

Kinoshita, H., Obata, S., Nasu, D., Kadota, K., Matsuo, T., \& Fleisig, G.S. (2017). Finger forces in fastball baseball pitching. Human Movement Science. 54, 172-181. https://doi.org/10.1016/j.humov.2017.04.007.

Kos, A., Milutinović, V., \& Umek, A. (2019). Challenges in wireless communication for connected sensors and wearable devices used in sport biofeedback applications. Future Generation Computer Systems, 92, 582-592. https://doi.org/10.1016/j.future.2018.03.032.

Kos, A., \& Umek, A. (2018a). Biomechanical Biofeedback Systems and Applications. Cham: Springer International Publishing.

Kos, A., \& Umek, A. (2018b). Smart sport equipment: SmartSki prototype for biofeedback applications in skiing. Personal and Ubiquitous Computing, 22(3), 535-544. https://doi.org/10.1007/s00779-018-1146-1.

Kos, A., \& Umek, A. (2019). Wearable sensor devices for prevention and rehabilitation in healthcare: swimming exercise with real-time therapist feedback. IEEE Internet of Things Journal, 6(2), 13311341. https://doi.org/10.1109/JIOT.2018.2850664.

Lee, S., Kim, K., Kim, Y. H., \& Lee, S. (2017). Motion anlaysis in lower extremity joints during ski carving turns using wearable inertial sensors and plantar pressure sensors. 2017 IEEE International Conference on Systems, Man, and Cybernetics (SMC), pp. 695-698.

Lencioni, T., Carpinella, I., Rabuffetti, M., Marzegan, A., \& Ferrarin, M. (2019). Human kinematic, kinetic and EMG data during different walking and stair ascending and descending tasks. Scientific data, 6(1), 309. https://doi.org/10.1038/s41597-019-0323-z

Mc Master, D. T. (2014). A brief review of strength and ballistic assessment methodologies in sport. Sports Med, 44(5), 603-23. 
Merletti, R., \& Muceli, S. (2019). Tutorial. Surface EMG detection in space and time: best practices. Journal Electromyography and Kinesiology, 49:p. 102363. https://doi.org/10.1016/j.jelekin.2019.102363.

Nigg, B.M. (2001). The role of impact forces and foot pronation: A new paradigm. Clinical Journal of Sport Medicine 11, 2-9.

Norris, M. (2014). Method analysis of accelerometers and gyroscopes in running gait: A systematic review. Curr Sports Med Rep, 8(3), 136-41.

Portnova-Fahreeva, A.A., Rizzoglio, F., Nisky, I., Casadio, M., Mussa-Ivaldi, F.A., \& Rombokas, E. (2020). Linear and Non-linear Dimensionality-Reduction Techniques on Full Hand Kinematics. Frontiers in bioengineering and biotechnology, 8, 429. https://doi.org/10.3389/fbioe.2020.00429

Purevsuren, T., Khuyagbaatar, B., Kim, K., \& Kim, Y.H. (2018). Investigation of knee joint forces and moments during short-track speed skating using wearable motion analysis system. International Journal of Precision Engineering and Manufacturing, 19(7):1055-1060.

Samozino, P., Horvais, N., \& Hintzy, F. (2007). Why does power output decrease at high pedaling rates during sprint cycling? Med Sci Sports Exerc., 39: 680-687.

Sanders, R.H., Gonjo, T., \& McCabe, C.B. (2015) Reliability of three-dimensional linear kinematics and kinetics of swimming derived from digitized video at 25 and $50 \mathrm{~Hz}$ with 10 and 5 frame extensions to the 4th order Butterworth smoothing window. Journal of Sports Science and Medicine 14, 441-451.

Sanders, R. H., Gonjo, T., \& McCabe, C. B. (2016). Reliability of Three-Dimensional Angular Kinematics and Kinetics of Swimming Derived from Digitized Video. Journal of sports science \& medicine, 15(1), 158-166.

Santello, M., Flanders, M., \& Soechting, J.F. (1998). Postural hand synergies for tool use. J. Neurosci. 18, 10105-10115. https://doi.org/10.1523/JNEUROSCI.18-23-10105.1998

Saponara, S. (2017). Wearable biometric performance measurement system for combat sports. IEEE Transactions on Instrumentation and Measurement, 66(10), 2545-2555. https://doi.org/10.1109/TIM.2017.2677679.

Sopa, I.S. (2014). Study regarding group cohesion at primary school level. Buletin of the Transilvania University of Braşov, 7.1(56), 67-74.

Sopa, I.S. (2019a). The influence of external factors in the efficiency of basketball scoring. Bulletin of the Transilvania University of Brasov, 12(1), 137-144.

Sopa, I.S. (2019b). Developing attack point in volleyball game using plyometric exercises at 13-14 years old volleyball players. Bulletin of the Transilvania University of Brasov, 12(2), 67-76.

Sopa, I.S., Neagu, N., \& Gliga, C.A. (2019). Correlation between body mass index and the apparition of spine, knee and feet deficiency in youth population. International Journal of Applied Exercise Physiology, 9(1), 8-15.

Sopa, I. S. (2015). Testing agility skill at a basketball team. Discobolul, Physical Education, Sport and Kinetotheraphy Journal, 9(42), 101-108.

Szabo, D.A., Neagu, N., Teodorescu, S., Pomohaci, M., \& Sopa, I.S. (2019). Does smart electronic devices influence the body deficiencies development at kids who practice swimming? International Journal of Applied Exercise Physiology, 8(2.1), 798-803.

Szabo, D.A., Neagu, N., \& Sopa, I.S. (2020). Kinematic angular analysis of cinematic biomechanics in forearm flexion: a case study. Geosport for Society, 13(2), 131-139. https://doi.org/10.30892/gss.130xx-065

Taborri, J., Keogh, J., Kos, A., Santuz, A., Umek, A., Urbanczyk, C., van der Kruk, E., \& Rossi, S. (2020). Sport Biomechanics Applications Using Inertial, Force, and EMG Sensors: A Literature Overview. Applied bionics and biomechanics, 2041549. https://doi.org/10.1155/2020/2041549

Taborri, J., Palermo, E., Rossi, S., \& Cappa, P. (2016). Gait partitioning methods: a systematic review. Sensors, 16(1), 66. https://doi.org/10.3390/s16010066.

Todorov, E., Ghahramani, Z. (2004). Analysis of the synergies underlying complex hand manipulation," in The 26th Annual International Conference of the IEEE Engineering in Medicine and Biology Society (San Francisco, CA: IEEE; ), 4637-4640.

Umek, A., Kos, A. (2016). The role of high-performance computing and communication for real-time biofeedback in sport. Mathematical Problems in Engineering; 11. https://doi.org/10.1155/2016/4829452. 
Urbanczyk, C.A., Mcgregor, A.H., \& Bull, A.M.J. (2019). Modelling scapular biomechanics to enhance interpretation of kinematics and performance data in rowing. Proceedings of the 37th International Conference of Biomechanics in Sports, 1-4.

van der Kruk, E., \& Reijne, M.M. (2018). Accuracy of human motion capture systems for sport applications; state-of-the-art review. European Journal of Sport Science, 18(6):806-819. doi: 10.1080/17461391.2018.1463397.

van der Kruk, E., Reijne, M.M., de Laat, B., \& Veeger, D.J. (2018). Push-off forces in elite short-track $\begin{array}{llll}\text { speed skating. } & \text { Sport }\end{array}$ https://doi.org/10.1080/14763141.2018.1441898.

van der Kruk, E., van der Helm, F.C.T., Veeger, H.E.J., \& Schwab, A.L. (2018). Power in sports: a literature review on the application, assumptions, and terminology of mechanical power in sport research. Journal of Biomechanics, 79, 1-14. https://doi.org/10.1016/j.jbiomech.2018.08.031.

Wakeling, J.M., Blake, O.M., \& Chan, H.K. (2010). Muscle coordination is key to the power output and mechanical efficiency of limb movements. J Exp Biol., 213, 487-492.

Whittlesey S.N., \& Robertson D.G. (2004) Two dimensional inverse dynamics. Research Methods in Biomechanics. Eds: Robertson D.G.E., et al. Chapter 5. Champaign, IL: Human Kinetics; 103.

Winter, D. A. (1991). Biomechanics and motor control of human gait: normal, elderly and pathological. 